\title{
Bernard Frank, Amour, colère, couleur. Essais sur le bouddhisme au Japon
}

Paris, Collège de France, Institut des hautes études japonaises, 2000, 280 p. (Préface de Claude Lévi-Strauss) (index, illustr., table des caract. chinois) (coll. " Bibliothèque de l'Institut des hautes études japonaises »)

\section{(2) OpenEdition}

\section{Édition électronique}

URL : http://journals.openedition.org/assr/588

DOI : $10.4000 /$ assr.588

ISSN : $1777-5825$

Éditeur

Éditions de l'EHESS

\section{Édition imprimée}

Date de publication : 1 octobre 2002

Pagination : 63-126

ISBN : 2-222-96725-2

ISSN : 0335-5985

Référence électronique

Jean-Pierre Berthon, "Bernard Frank, Amour, colère, couleur. Essais sur le bouddhisme au Japon »,

Archives de sciences sociales des religions [En ligne], 120 | octobre - décembre 2002, document 120.14 mis en ligne le 17 octobre 2005, consulté le 21 septembre 2020. URL : http://journals.openedition.org/ assr/588 ; DOI : https://doi.org/10.4000/assr.588 
ment le lecteur à se faire une idée sur ces questions complexes.

Dans le chapitre sur l'Indonésie «L'islam en Asie du Sud-est: le legs religieux de la course aux épices » (pp. 145-206), A.F. réussit l'exploit de présenter au lecteur un tableau global de la situation, malgré l'extraordinaire complexité géographique, démographique et religieuse de cette partie du monde. S'y ajoute une analyse de l'impact européen, avec la formalisation du droit (sous l'administration britannique d'abord, puis sous l'administration hollandaise), sans oublier les mouvements de réforme islamique. La partie consacrée à l'islam politique au $\mathrm{XX}^{\mathrm{e}}$ siècle est traitée tour à tour: en Indonésie, en Malaisie, à Singapour, aux Philippines, en Birmanie, en Thaïlande, à Bruneï et au Cambodge. Puis 1'A. aborde les questions de pratiques religieuses, du statut de la femme et de l'éducation, en Indonésie et en Malaisie. Le chapitre se termine par une bibliographie sommaire (particulièrement bienvenue dans ce cas !) et une chronologie (allant de 1292 à 2001).

Le chapitre sur l'islam en Chine, d'Élisabeth Allès «Confucius, Allah et Mao » (pp. 207239, avec une bibliographie et une chronologie allant de 651 à 1989), décrit brillamment, de façon synthétique et avec beaucoup de clarté, l'histoire - plus que millénaire - de l'islam en Chine, dont les adeptes (Hui à l'Est, Ouighours du Xinjang ou du Turkestan oriental à l'Ouest), très largement minoritaires, revendiquent néanmoins le droit d'avoir leur propre vision de leur histoire et de leur culture (avec, à la clé, le problème crucial et bien connu dans tous les pays communistes, celui de la reconnaissance officielle par les autorités de leur groupe « ethnique »). Se greffent naturellement les influences, plus ou moins bien perçues, venant du monde musulman extérieur, les diverses variantes du soufisme, des réformismes et des « islams politiques » divers, sans parler des contraintes imposées par les autorités du pays, non musulmanes évidemment.

Le volume se termine par deux annexes (glossaire des termes utilisés, et liste des cartes, qui aident à se retrouver dans ces lointaines contrées). Ouvrage extraordinaire, comme il n'en a jamais existé auparavant, qui nous renseigne de façon magistrale sur plus de la moitié des musulmans du monde!

Alexandre Popovic.
FRANK (Bernard).

Amour, colère, couleur. Essais sur le bouddhisme au Japon. Paris, Collège de France, Institut des hautes études japonaises, 2000, 280 p. (Préface de Claude Lévi-Strauss) (index, illustr., table des caract. chinois) (coll. « Bibliothèque de l'Institut des hautes études japonaises »).

Faisant suite à la publication des résumés annuels des cours de B.F. au Collège de France - Dieux et Bouddhas au Japon. Travaux du Collège de France, Paris, Éditions Odile Jacob, 2000 -, paraissait la même année l'ouvrage complémentaire qui allait permettre à un public moins spécialisé, mais tout autant exigeant, d'en apprécier tous les développements. C'est une nouvelle fois au dévouement et à l'abnégation des collègues et amis de B.F. que nous sommes redevables de la parution de ces essais sur les divers aspects du bouddhisme japonais, en un temps si court. Se trouve ici rassemblé un ensemble, jusqu'alors disséminé, de textes et de conférences, pour certains déjà publiés et pour d'autres restés inédits, qui renseigne également, avec bonheur, sur le parcours et les choix intellectuels qui furent ceux de l'auteur tout au long de sa vie.

L'ouvrage s'ouvre sur une "synthèse » du bouddhisme à travers trois thèmes chers à B.F. : la présence d'un être vénéré dans ses images et le problème de l'être vénéré lui-même; la métaphore végétale du tronc bouddhique et de ses ramifications, résumée dans la formule de la «double vérité du Buddha»; la rencontre, enfin, du bouddhisme avec la littérature de la période de Heian (794-1185), époque qu'il affectionnait tout particulièrement. À l'intérieur de chacun de ces thèmes, après avoir exposé le cheminement de la pensée bouddhique, B.F. ne manque pas de s'interroger sur la réalité actuelle ou populaire du phénomène décrit. Dissertant, ainsi, sur l'image que se faisaient du bouddhisme les Japonais de Heian, l'A. écrit : «On ne manquera pas de se demander si une telle image correspond bien à celle qu'avait la population en général et si nous ne venons pas de décrire des conceptions qui n'appartenaient qu'à l'aristocratie. Celle-ci n'était-elle pas la seule encore, en ce temps, à avoir été réellement touchée par le bouddhisme ?». Ce souci de l'exhaustivité et cette interrogation constante quant aux possibles généralisations, se déploient tout au long des études qui forment la seconde partie de l'ouvrage. Ces études concernent principalement l'histoire et l'iconographie, à la fois savante et populaire, d'une dizaine de divinités du panthéon bouddhique avec, pour certaines d'entre elles, un véritable travail 
d'enquête qui est aussi l'une des marques caractéristiques du travail de B.F., comme le souligne Claude Lévi-Strauss dans la préface de l'ouvrage: "Parcourant le pays, enquêtant dans les temples, déchiffrant [...] des symboles oubliés dans les images pieuses distribuées aux fidèles, curieux de littérature populaire autant que de philosophie, B.F. entraîne le lecteur dans des aventures intellectuelles passionnantes et pleines d'inattendu. »

Ces aventures ne sont jamais le fruit du hasard. Elles sont l'aboutissement d'un long cheminement qui passe par une érudition sans faille et une curiosité qui ont fait de B.F. un arpenteur inlassable de l'archipel. C'est pourquoi les ethnologues, tout autant que les historiens des religions, trouveront une mine de renseignements, pris aux sources les plus diverses, sur les avatars des divinités présentées, sur leurs processus adaptatifs et sur la transformation des cultes qui leur ont été rendus au cours de l'histoire japonaise. Mais la véritable assise sur laquelle repose les interprétations savantes de B.F. s'appuie sur l'examen, sans cesse renouvelé, du syncrétisme religieux japonais; c'est-à-dire de l'amalgame opéré entre le bouddhisme, sous ses formes variées, et la multitude de cultes locaux avec lesquels il entra en contact. C'est à l'encontre de ce syncrétisme toujours vivant, en dépit de la tentative d'éradication du bouddhisme à l'époque Meiji, que B.F. se plaît à déployer ses efforts pour nous en faire comprendre toute la complexité, offrant ainsi aux comparatistes un nouveau terrain d'investigation.

Les réflexions conclusives que B.F. expose à la fin d'une présentation du «dieu-roi » Bishamon-ten, résument parfaitement sa méthode : « J'espère vous avoir conduits à penser que, dans une recherche d'une telle nature, il est nécessaire de faire appel à toutes sortes de documents écrits, oraux ou figurés, des plus apparemment "sérieux » aux plus familiers, des plus anciens aux plus contemporains, des plus généraux à ceux qui sont de simple diffusion locale - cela à condition, bien sûr, de savoir les situer dans leur contexte et d'en faire la critique. » (p. 121)

Malgré les soixante-huit illustrations reproduites en fin de volume, nombre de divinités décrites et analysées par l'A. n'ont pu, pour des raisons éditoriales et à cause de l'absence de l'A., être jointes au texte. Il reste, heureusement, les références bibliographiques qui permettent de combler cette lacune.

Jean-Pierre Berthon.
Christians in Asia Before 1500. Richmond Surrey, Curzon, 1999, 391 p. (bibliogr., cartes, index).

Ce livre sur l'histoire prémoderne des communautés chrétiennes d'Asie est né du constat que les chrétiens d'Occident ignoraient généralement tout au sujet de ces dernières et que cette ignorance était largement partagée par les intéressées, mal informées sur les chrétientés asiatiques du passé. Il embrasse les quatorze siècles d'histoire qui vont des débuts du christianisme à l'émergence des empires coloniaux occidentaux et couvre un espace lui aussi très vaste. De son titre l'Asie est en effet à prendre dans son sens géographique le plus large puisque y sont englobées les régions situées entre le lieu de naissance du christianisme l'Israël moderne - et la Chine, en passant par l'Arabie, la Syrie, l'Arménie et la Géorgie, la Perse, l'Afghanistan et l'Asie centrale, l'Inde et l'Asie du Sud-Est. Étant donné l'étendue géographique couverte et la durée chronologique concernée, il n'offre pas du neuf dans tous les domaines, mais il comble pourtant un vide en présentant une synthèse intelligente et parfaitement maîtrisée de l'ensemble des connaissances accumulées, y compris en tenant compte des travaux les plus récents et les plus difficilement accessibles car disséminés dans des journaux de recherche à faible diffusion.

L'ouvrage répartit sa matière en 12 chapitres. Les trois premiers ont une portée générale, les suivants sont consacrés chacun à une communauté chrétienne particulière. Une brève conclusion termine l'ensemble.

Après une introduction qui précise les intentions des auteurs, le deuxième chapitre présente les éléments scripturaux et théoriques indispensables pour s'y retrouver dans les différentes successions apostoliques qui jouèrent un rôle central dans l'organisation des Églises chrétiennes de l'Occident comme de l'Asie, chaque Église mettant en avant l'autorité supérieure de son fondateur pour se légitimer. Les A.A. donnent une bonne idée des enjeux qui s'attachent à la revendication d'une fondation apostolique à travers l'exemple des chrétiens de saint Thomas (Inde). Ce choix est d'autant plus parlant que la question des origines garde toute son actualité pour cette communauté chrétienne qui, à la différence d'autres communautés anciennes d'Asie, a survécu jusqu'à nos jours. Il y a deux thèses en présence : selon l'une, l'apôtre Thomas a évangélisé l'Inde, selon l'autre, aucune preuve historique ne permet de l'affirmer. Mais, comme le soulignent les A.A., on ne saurait en conclure 